\title{
Direct Enhancement of Nuclear Singlet Order by Dynamic Nuclear Polarization
}

\author{
Michael C. D. Tayler, ${ }^{\dagger}$ Irene Marco-Rius, ${ }^{\ddagger, \S}$ Mikko I. Kettunen, ${ }^{\ddagger}$, Kevin M. Brindle, $^{\ddagger}$, \\ Malcolm H. Levitt, ${ }^{* \dagger}$ and Giuseppe Pileio ${ }^{\dagger} \dagger$ \\ ${ }^{\dagger}$ School of Chemistry, Southampton University, Southampton SO17 1BJ, U.K. \\ ${ }^{\ddagger}$ Department of Biochemistry, Cambridge University, Tennis Court Road, Cambridge CB2 1GA, U.K. \\ ${ }^{\S}$ Cancer Research UK Cambridge Research Institute, Robinson Way, Cambridge CB2 ORE, U.K.
}

ABSTRACT: Hyperpolarized singlet order is available immediately after dissolution DNP, avoiding need for additional preparation steps. We demonstrate this procedure on a sample of $\left[1,2-{ }^{13} \mathrm{C}_{2}\right]$ pyruvic acid.

$\mathrm{T}_{\mathrm{p}}^{\mathrm{h}}$ he large signal improvement provided by spin hyperpolarization methods has dramatically extended the in vivo potential of NMR spectroscopy. ${ }^{1}$ Hyperpolarized nuclear spin order permits tracing of both endogenous and nonendogenous substances as they are transported through the blood vessels and organs and participate in metabolism. ${ }^{2-4} \mathrm{~A}$ limitation of these techniques is the often short observation time scale imposed by nuclear spin relaxation. Hyperpolarized magnetization typically decays with the longitudinal relaxation time $T_{1}$, which is typically between a fraction of a second and 1 min and can be particularly short in vivo because of the presence of paramagnetic species and high concentrations of other magnetic nuclei. Short decay times hinder the use of hyperpolarized substrates for imaging metabolism in vivo because of the relatively long times taken for substrates to reach the tissue of interest from the point of injection. For instance, while hyperpolarized ${ }^{129} \mathrm{Xe}$ can be used as a lung imaging agent in gas-phase magnetic resonance imaging (MRI) because its relaxation time is several hours in the gas phase, its potential as a tracer in blood is very limited because the $T_{1}$ relaxation time of ${ }^{129} \mathrm{Xe}$ is reduced to only a few seconds upon dissolution. ${ }^{5}$

A potential remedy for the limited hyperpolarization lifetime is the use of nuclear singlet states involving coupled spin- $-1 / 2$ pairs. The lifetime of the nuclear singlet state $(|\alpha \beta\rangle-|\beta \alpha\rangle) /$ $\sqrt{2}$ is capable of exceeding $T_{1}$ in some circumstances, since many common relaxation mechanisms are symmetric with respect to spin exchange and cannot induce singlet-triplet transitions. $^{6-9}$ In previous work, we reported the potential of doubly ${ }^{15} \mathrm{~N}$-labeled nitrous oxide, ${ }^{15} \mathrm{~N}_{2} \mathrm{O}$. ${ }^{10,11}$ The ordinary $T_{1}$ lifetime of the spin order is $30 \mathrm{~s}$ for ${ }^{15} \mathrm{~N}_{2} \mathrm{O}$ dissolved in human blood. The singlet decay constant in blood, $\sim 7 \mathrm{~min}$, is 15 times longer. ${ }^{11}$

In some cases, substances exhibiting hyperpolarized singlet order are accessible directly by chemical reactions of hydrogen enriched with the para-hydrogen spin isomer. ${ }^{12-14}$ An alternative approach is to prepare hyperpolarized magnetization using a method such as dynamic nuclear polarization $(\mathrm{DNP})^{15,16}$ and then to convert it into singlet order. Several conversion methods are available:
- application of resonant radiofrequency pulses in a high magnetic field to excite a "precursor state", 7 which is transformed into singlet order upon adiabatic transport to a low field; ${ }^{17,18}$

- preparation of singlet order in a high magnetic field using a radiofrequency pulse sequence; ${ }^{19-21}$

- application of an audio-frequency pulse sequence in a low magnetic field on a prepolarized sample; ${ }^{22}$

- exploitation of chemical symmetry-switching reactions. ${ }^{23}$

All of these rely upon manipulations in addition to spin hyperpolarization. In certain cases they may be difficult to implement, require extra hardware, or take up valuable time during an experiment.

In this communication, we show that these preparations may be circumvented by the availability of singlet spin order in the initial hyperpolarized state of coupled $S=1 / 2$ spins. This demonstrates "direct" access (i.e., without extra manipulation of the sample) to hyperpolarized spin order that is potentially longer-lived than $T_{1}$. The following is a description of singlet order obtained using the dissolution DNP technique, ${ }^{15}$ but the principle should apply to other nuclear hyperpolarization methods as well.

The polarization $p$ of an ensemble of noninteracting $S=1 / 2$ spins is defined by

$$
p=\left(n_{\alpha}-n_{\beta}\right)
$$

where $n_{\alpha}$ and $n_{\beta}$ are the populations of the spin states with angular momentum parallel and antiparallel to the magnetic field, respectively, normalized so that $n_{\alpha}+n_{\beta}=1$. The populations are therefore given in terms of the polarization by $n_{\alpha}=(1+p) / 2$ and $n_{\beta}=(1-p) / 2$. The thermal-equilibrium polarization $p_{\text {thermal }}$ at temperature $T$ and a magnetic field strength $B^{0}$ is given through the Boltzmann model by the expression $p_{\text {thermal }}=\tanh \left(\hbar \gamma_{\text {nuc }} B^{0} / 2 k_{\mathrm{B}} T\right)$, where $\gamma_{\text {nuc }}$ is the gyromagnetic ratio of the nucleus, $\hbar$ is Planck's constant divided by $2 \pi$, and $k_{\mathrm{B}}$ is Boltzmann's constant. For ${ }^{13} \mathrm{C}$, this is on the order of $10^{-5}$ at room temperature in the typical working field of an in vivo MRI magnet $(\sim 4.7 \mathrm{~T})$. For hyperpolarized samples, $p \gg p_{\text {thermal }}$; the limiting situation is $|p|$ $=1$, which corresponds to complete polarization along the quantization axis. This represents the maximum population asymmetry $n_{\alpha}-n_{\beta}$ and the maximum sample magnetization.

Received: April 2, 2012

Published: April 18, 2012 
In the case of chemically inequivalent $\operatorname{spin}^{-1} / 2$ pairs in a strong magnetic field, the energy eigenstates are given by product states of the form $\left|\alpha_{1} \alpha_{2}\right\rangle=\left|\alpha_{1}\right\rangle\left|\alpha_{2}\right\rangle,\left|\alpha_{1} \beta_{2}\right\rangle=\left|\alpha_{1}\right\rangle\left|\beta_{2}\right\rangle$, etc., for the coupled angular momenta of the nuclei. The populations of these states are simply products of those for the two nuclei:

$$
\begin{aligned}
& n_{\alpha_{1} \alpha_{2}}=(1+p)(1+p) / 4 \\
& n_{\alpha_{1} \beta_{2}}=(1-p)(1+p) / 4 \\
& n_{\beta_{1} \alpha_{2}}=(1+p)(1-p) / 4 \\
& n_{\beta_{1} \beta_{2}}=(1-p)(1-p) / 4
\end{aligned}
$$

Longitudinal polarization in this case is the population difference $n_{\alpha_{1} \alpha_{2}}-n_{\beta_{1} \beta_{2}}$, which again equals $p$.

Singlet order proportional to $p^{2}$ is generated from an imbalance between the populations $n_{\alpha_{1} \beta_{2}}+n_{\beta_{1} \alpha_{2}}$ and $n_{\alpha_{1} \alpha_{2}}+n_{\beta_{1} \beta_{2}}$. In the dissolution DNP experiment, ${ }^{15}$ the process occurs as follows: Nuclear polarization $p \approx p_{\mathrm{e}} \gg p_{\text {thermal }}$ is obtained in a high magnetic field at a temperature of $\sim 1 \mathrm{~K}$ through contact with a strongly polarized electron bath $\left[p_{\mathrm{e}} \approx \tanh \left(\hbar \gamma_{\mathrm{e}} B^{0} / 2 k_{\mathrm{B}} T\right)\right.$ $\approx 1$ ] in the presence of off-resonance microwave irradiation close to the electron Larmor frequency. The frozen sample is dissolved with a jet of hot solvent and collected as a roomtemperature liquid in a region of low magnetic field outside the polarizer. During this transport, the high-field eigenstates are adiabatically transformed into the nuclear singlet $\left(\left|S_{0}\right\rangle\right)$ and triplet $\left(\left|T_{M}\right\rangle\right)$ energy eigenstates in the low magnetic field, which are defined as follows:

$$
\begin{aligned}
\left|S_{0}\right\rangle & =\left(\left|\alpha_{1} \beta_{2}\right\rangle-\left|\beta_{1} \alpha_{2}\right\rangle\right) / \sqrt{2} \\
\left|T_{0}\right\rangle & =\left(\left|\alpha_{1} \beta_{2}\right\rangle+\left|\beta_{1} \alpha_{2}\right\rangle\right) / \sqrt{2} \\
\left|T_{+1}\right\rangle & =\left|\alpha_{1} \alpha_{2}\right\rangle \\
\left|T_{-1}\right\rangle & =\left|\beta_{1} \beta_{2}\right\rangle
\end{aligned}
$$

For a weakly coupled spin pair with positive spin-spin coupling, gyromagnetic ratio, and chemical shift difference $\delta_{1}$ $-\delta_{2}$, the correspondence between the low-field and high-field states is ${ }^{14,24}$

$$
\begin{gathered}
\left|\alpha_{1} \beta_{2}\right\rangle \rightarrow\left|S_{0}\right\rangle \quad\left|\beta_{1} \alpha_{2}\right\rangle \rightarrow\left|T_{0}\right\rangle \\
\left|\alpha_{1} \alpha_{2}\right\rangle \rightarrow\left|T_{+1}\right\rangle \quad\left|\beta_{1} \beta_{2}\right\rangle \rightarrow\left|T_{-1}\right\rangle
\end{gathered}
$$

Nuclear singlet order corresponds to the mean singlet-triplet population difference. If relaxation losses during transport are neglected, this is given by

$$
\begin{aligned}
p_{S} & =n_{\alpha_{1} \beta_{2}}-\left(n_{\alpha_{1} \alpha_{2}}+n_{\beta_{1} \alpha_{2}}+n_{\beta_{1} \beta_{2}}\right) / 3 \\
& =-p^{2} / 3
\end{aligned}
$$

The negative sign arises since strong polarization leads to an excess population in the triplet state, depleting the singlet state. Hyperpolarized singlet order is therefore available immediately after dissolution, albeit with significantly less intensity than the longitudinal order. The singlet polarization may nevertheless still be substantial compared to $p_{\text {thermal }}$. For instance, a $30 \%$ polarized ensemble $(p=0.3)$ should yield $3 \%$ negative singlet order $\left(p_{\mathrm{S}}=-0.03\right)$, which is 3 orders of magnitude larger than the thermal polarization.
In a low magnetic field, the singlet order decays with a time constant $T_{S}$, which in suitable cases is longer than the magnetization relaxation time $T_{1}$. The long singlet lifetime may be exploited by introducing the singlet-hyperpolarized molecules into an object of interest. Apart from singlet relaxation, the singlet order persists as long as the subject remains in the low field and the bond connecting the spincoupled nuclei remains intact.

Eventual conversion of singlet order to an NMR observable is done by transporting the sample back into a high magnetic field, following the reverse of the transformations in eq 4 (Figure 1a). ${ }^{14}$ The resulting singlet postcursor ${ }^{7}$ state corre-

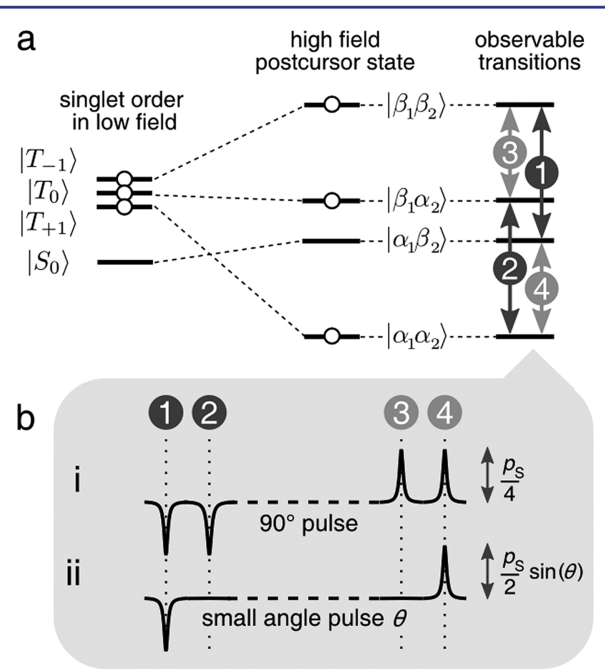

Figure 1. (a) Correlation between singlet population order in low magnetic field and the adiabatic postcursor state on which NMR readout is performed. The population distribution assumes a pure singlet polarization $p_{\mathrm{S}}=-{ }^{1} / 3$. Labels apply to the case $\left(\delta_{1}-\delta_{2}\right)>0, \gamma$ $>0$, and $J>0$. (b) Schematic spectra generated from pure singlet $\operatorname{order} p_{\mathrm{S}}$ using (i) a strong 90 degree read pulse; (ii) a pulse of small flip angle $\theta$, in the limit $|\sin (\theta)| \ll 1$.

sponds to a population deficit in $\left|\alpha_{1} \beta_{2}\right\rangle$ and equal populations in the other states. A strong, nonselective radiofrequency pulse generates an NMR signal that comprises the outer doublet peaks with opposite amplitudes given by

$$
\pm \exp \left(-t / T_{\mathrm{S}}\right) \times p_{\mathrm{S}} \times \cos ^{2}(\theta / 2) \sin (\theta) / 2
$$

for a pulse flip angle $\theta$ and low-field waiting time $t$, and inner doublet peaks with opposite amplitudes given by

$$
\pm \exp \left(-t / T_{\mathrm{S}}\right) \times p_{\mathrm{S}} \times \sin ^{2}(\theta / 2) \sin (\theta) / 2
$$

Figure $1 \mathrm{~b}$ illustrates the spectra in the case of (i) a $90^{\circ}$ pulse $(\theta$ $=\pi / 2$ ) and (ii) a small flip angle pulse $(|\theta| \ll \pi / 2)$. The small flip angle gives peak intensities proportional to the population differences across the states. In this case, the two outer peaks occur with opposite amplitudes that are proportional to $p^{2} / 3$. The inner transitions are absent because the singlet postcursor contains equal populations in the connected states.

A nonselective pulse applied to pure longitudinal order excites all transitions with an equal amplitude given by

$$
\exp \left(-t / T_{1}\right) \times p \times \sin (\theta) / 4
$$

The singlet order therefore results in a spectrum where the area under each $J$ doublet is $\left(p_{S} / p\right) \exp \left[\left(1 / T_{1}-1 / T_{S}\right) t\right]$ times that 
from the longitudinal magnetization, after the same time in the low field and the same pulse flip angle.

A natural target for hyperpolarization of singlet order is ${ }^{13} \mathrm{C}_{2}$ labeled pyruvate (2-oxo $\left[1,2-{ }^{13} \mathrm{C}_{2}\right]$ propanoic acid), which is used for simultaneous real-time monitoring of pyruvate dehydrogenase and Krebs cycle metabolism in heart. ${ }^{25}$ According to a previously described procedure, ${ }^{4}$ labeled pyruvic acid was polarized in an $\alpha$-prototype hyperpolarizer (GE Healthcare, Amersham, U.K.) working at 3.35 T. A mixture containing $43.5 \mathrm{mg}$ of $\left[1,2-{ }^{13} \mathrm{C}_{2}\right]$ pyruvic acid (95\% purum; Sigma-Aldrich, Dorset, U.K.), $0.7 \mathrm{mg}$ of the trityl radical OX063 (GE Healthcare, Little Chalfont, U.K.), and $1.2 \mathrm{mg}$ of $0.1 \mu \mathrm{L}$ gadolinium chelate solution (gadoteric acid, Dotarem; Guerbet, Roissy, France) was cooled to $1.2 \mathrm{~K}$ in liquid $\mathrm{He}$ and irradiated with a $100 \mathrm{~mW}$ microwave source at $\sim 94 \mathrm{GHz}$. The buildup of ${ }^{13} \mathrm{C}$ polarization was monitored via the solid-state NMR signal. After $\sim 1 \mathrm{~h}$ of microwave irradiation, the frozen material was dissolved with a jet of hot buffer solution $[6 \mathrm{~mL}$, heated to $180{ }^{\circ} \mathrm{C}$, containing $100 \mathrm{mg} / \mathrm{L}$ EDTA, $30 \mathrm{mM} \mathrm{NaCl}$, $94 \mathrm{mM} \mathrm{NaOH}$, and $40 \mathrm{mM}$ 4-(2-hydroxyethyl)-1-piperazineethanesulfonic acid (HEPES)], and the solution was collected in a vial located outside the magnet.

To estimate the amplitudes of the longitudinal polarization and the singlet order, the hyperpolarized solution was divided in equal portions between two identical $10 \mathrm{~mm}$ o.d. NMR sample tubes, each already containing $2.0 \mathrm{~mL}$ of $\mathrm{D}_{2} \mathrm{O}$. The final pyruvate concentration in each tube was $\sim 10 \mathrm{mM}$. The first tube was inserted immediately into a high-field NMR spectrometer (9.4 T, Varian Inova), and the ${ }^{13} \mathrm{C}$ spectrum was recorded after a $6^{\circ}$ flip angle pulse. The spectrum, which is shown in Figure 2a, is characteristic of the predominant longitudinal spin order.

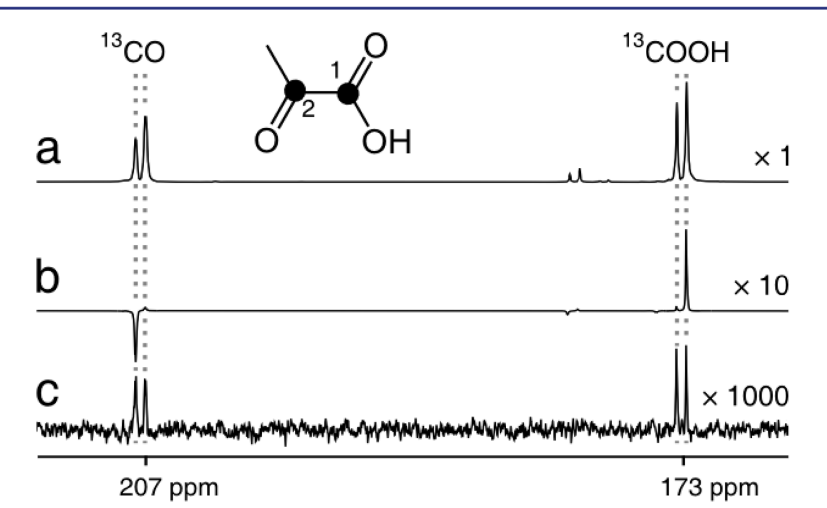

Figure $2 .{ }^{13} \mathrm{C}$ NMR spectra at $9.4 \mathrm{~T}$ using a $6{ }^{\circ}$ flip angle for detection recorded (a) on hyperpolarized $\left[1,2-{ }^{13} \mathrm{C}_{2}\right]$ pyruvate (single scan); (b) after erasing the magnetization by shaking inside a mu-metal chamber followed by transfer into a high field (single scan); and (c) later on the same sample as in (a), thermally polarized (16 scans).

The second tube, meanwhile, was inserted into a mu-metal cylinder and shaken for $\sim 5 \mathrm{~s}$. This sample was then removed from the cylinder and inserted into the NMR spectrometer, and the spectrum was taken using a $6^{\circ}$ flip angle pulse. The shaking of the sample in the weak and sharply inhomogeneous magnetic field in the interior of the mu-metal chamber had the effect of rapidly equilibrating the nuclear triplet populations while leaving the singlet order intact, since the scalar singlet order is invariant to rotations. The shaking in the mu-metal chamber therefore left only a population asymmetry between the singlet and triplet states. The spectrum shown in Figure $2 b$ displays the pattern predicted in Figure $1 \mathrm{~b}(\mathrm{ii})$, demonstrating the presence of significant singlet order in the low magnetic field. The presence of singlet order is also indicated through the asymmetry in the doublet peak components in Figure 2a.

By comparing the integral of the spectrum of the first tube with that of the same sample at thermal equilibrium (Figure 2c), we obtained an estimated longitudinal polarization $p$ of 26 $\pm 1 \%$ at the time of arrival in the detection magnet $(\sim 15 \mathrm{~s}$ after dissolution). If it is assumed that there was no relaxation of the sample during transport, this value of $p$ corresponds to an expected singlet polarization of $2.3 \%$, which is roughly 12 times less than the longitudinal polarization. In our experiment, the signal from the singlet order (a further $15 \mathrm{~s}$ after dissolution; see Figure $2 \mathrm{~b}$ ) was 20 times weaker than that obtained for the first tube (Figure 2a), as deduced by comparing the integrals across the doublet at $173 \mathrm{ppm}$. This is reasonably consistent with the directly generated singlet order being $p / 3$ times the longitudinal magnetization. The factor of 2 discrepancy is attributed to the relaxation during the additional low-field manipulations involved in the singlet NMR experiment.

Another experiment was performed to estimate the decay constant $T_{\mathrm{S}}$ for the $\left[1,2-{ }^{13} \mathrm{C}_{2}\right]$ pyruvate at low field $(\sim 0.5 \mathrm{mT})$. After exiting the hyperpolarizer, the pyruvate solution was shaken in the mu-metal cylinder and then diluted upon being added to a vial containing $15 \mathrm{~mL}$ of $\mathrm{D}_{2} \mathrm{O}$. This solution was pipetted equally into six $10 \mathrm{~mm}$ o.d. NMR tubes, which were then inserted at $30 \mathrm{~s}$ intervals into the high-field spectrometer, where a small flip angle spectrum was recorded. Figure 3 shows

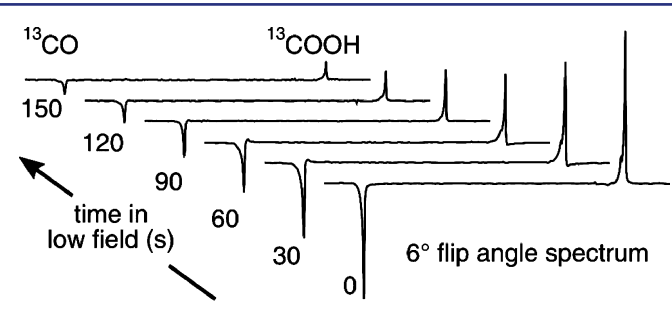

Figure 3. Decay of singlet order in $\left[1,2-{ }^{13} \mathrm{C}_{2}\right]$ pyruvic acid in the laboratory magnetic field. The fitted time constant was $T_{\mathrm{S}}=70 \pm 2 \mathrm{~s}$.

the spectra from the six tubes after the different low-field waiting times. The peak integrals were fit by an exponential decay $\exp \left(-t / T_{\mathrm{S}}\right)$, giving a decay constant $T_{\mathrm{S}}$ of $70 \pm 2 \mathrm{~s}$.

The value of $T_{1}$ for the same system was later measured on the thermally polarized sample using a field-cycled experiment. The sample was polarized to $p=p_{\text {thermal }}$ in the high-field spectrometer magnet and then shuttled outside the magnet to rest for a time in a low field $(0.5 \mathrm{mT})$ for relaxation. Upon reinsertion in the magnet, a $90^{\circ}$ pulse was applied and the NMR spectrum recorded. Spectra were recorded for different low-field waiting times, and the integrals were then fit to $\exp \left(-t / T_{1}\right)$, giving a decay constant $T_{1}$ of $36 \pm 1 \mathrm{~s}$ for both carbons.

These results show that the singlet order in $\left[1,2-{ }^{13} C_{2}\right]$ pyruvate persists twice as long as the conventional polarization in a low magnetic field. The $T_{S} / T_{1}$ ratio of only $\sim 2$, however, is slightly disappointing, since it indicates the presence of relaxation mechanisms in pyruvate that have a strong effect on singlet relaxation. The nature of the mechanism is currently under investigation; intermolecular dipole-dipole interactions and spin-rotation couplings are both possible candidates.

To summarize, we have demonstrated that singlet order is available "directly" from the initial hyperpolarized state of a 
sample containing coupled spin- ${ }^{1} / 2$ pairs. Although the singlet order achieved is significantly less than the longitudinal polarization, this route eliminates the additional hardware and complexity involved in other singlet preparation methods

\section{AUTHOR INFORMATION}

\section{Corresponding Author}

mhl@soton.ac.uk; g.pileio@soton.ac.uk

\section{Notes}

The authors declare no competing financial interest.

\section{ACKNOWLEDGMENTS}

Support was provided by EPSRC-UK, The Leverhulme Trust, the European Research Council, and Cancer Research UK. I.M.-R. acknowledges the European Union Seventh Framework Programme (FP7) and the Marie Curie Initial Training Network Program METAFLUX (Project 264780). We are also grateful to Brett Kennedy (Cambridge University) for experimental help.

\section{REFERENCES}

(1) Viale, A.; Aime, S. Curr. Top. Chem. Biol. 2010, 14, 90-96.

(2) Golman, K.; in't Zandt, R.; Lerche, M.; Pehrson, R.; ArdenkjærLarsen, J. H. Cancer Res. 2006, 66, 10855-10860.

(3) Gallagher, F.; Kettunen, M. I.; Brindle, K. M. Prog. NMR Spectrosc. 2009, 55, 285-295.

(4) Day, S. E.; Kettunen, M. I.; Gallagher, F. A.; Hu, D.-E.; Lerche, M.; Wolber, J.; Golman, J. H.; Ardenkjær-Larsen, K.; Brindle, K. M. Nat. Med. 2007, 13, 1382-1387.

(5) Möller, H. E.; Chen, X. J.; Saam, B.; Hagspiel, K.; Johnson, G. A.; Altes, T. A.; de Lange, E. E.; Kauczor, H.-U. Magn. Reson. Med. 2002, 47, 1029-1051.

(6) Levitt, M. H. Singlet and Other States with Extended Lifetimes. In Encyclopedia of Magnetic Resonance, Vol. 9; Wiley: Chichester, U.K., 2010.

(7) Levitt, M. H. Annu. Rev. Phys. Chem. 2012, 63, 89-105.

(8) Pileio, G. Prog. NMR Spectrosc. 2010, 56, 217-231.

(9) Tayler, M. C. D.; Levitt, M. H. Phys. Chem. Chem. Phys. 2011, 13, 9128-9130.

(10) Pileio, G.; Carravetta, M.; Hughes, E.; Levitt, M. H. J. Am. Chem. Soc. 2008, 130, 12582-12583.

(11) Ghosh, R. K.; Kadlecek, S. J.; Ardenkjær-Larsen, J. H.; Pullinger, B. M.; Pileio, G.; Levitt, M. H.; Kuzma, N. N.; Rizi, R. R. Magn. Reson. Med. 2011, 66, 1177-1180.

(12) Bowers, C. R.; Weitekamp, D. P. J. Am. Chem. Soc. 1987, 109, $5541-5542$.

(13) Bowers, C. R. Sensitivity Enhancement using Parahydrogen. In Encyclopedia of Magnetic Resonance; Wiley: Chichester, U.K., 2007.

(14) Pravica, M. G.; Weitekamp, D. P. Chem. Phys. Lett. 1988, 145, $255-258$.

(15) Ardenkjær-Larsen, J. H.; Fridlund, B.; Gram, A.; Hansson, G.; Hansson, L.; Lerche, M. H.; Servin, R.; Thaning, M.; Golman, K. Proc. Natl. Acad. Sci. U.S.A. 2003, 100, 10158-10163.

(16) Lingwood, M. D.; Han, S. Ann. Rep. NMR Spectrosc. 2011, 73, 83-126.

(17) Carravetta, M.; Johannessen, O. G.; Levitt, M. H. Phys. Rev. Lett. 2004, 92, No. 153003.

(18) Bornet, A.; Jannin, S.; Bodenhausen, G. Chem. Phys. Lett. 2011, $512,151-154$.

(19) Carravetta, M.; Levitt, M. H. J. Am. Chem. Soc. 2004, 126, $6228-6229$.

(20) Vasos, P. R.; Comment, A.; Sarkar, R.; Ahuja, P.; Jannin, S.; Ansermet, J. P.; Konter, J. A.; Hautle, P.; van den Brandt, B.; Bodenhausen, G. Proc. Natl. Acad. Sci. U.S.A. 2009, 106, 1846918473.
(21) Tayler, M. C. D.; Levitt, M. H. Phys. Chem. Chem. Phys. 2011, $13,5556-5560$

(22) Pileio, G.; Carravetta, M.; Levitt, M. H. Proc. Natl. Acad. Sci. U.S.A. 2010, 107, 17135-17139.

(23) Warren, W. S.; Jenista, E.; Branca, R. T.; Chen, X. Science 2009, 323, 1711-1714.

(24) Carravetta, M.; Levitt, M. H. J. Chem. Phys. 2005, 122, No. 214505.

(25) Chen, A. P.; Hurd, R. E.; Schroeder, M. A.; Lau, A. Z.; Gu, Y.-p.; Lam, W. W.; Barry, J.; Tropp, J.; Cunningham, C. H. NMR Biomed. 2012, 305-311. 